\title{
Detection of Retinal Hemorrhage in Color Fundus Image
}

\author{
Priyanka Kale ${ }^{1}$, Prof. N. Janwe ${ }^{2}$ \\ Department of Computer Technology, Rajiv Gandhi College of Engineering, Research \& Technology ${ }^{1,2}$
}

\begin{abstract}
Diabetic Retinopathy (DR) is an eye disease due to diabetes which causes visual loss. So it is reasonable to provide treatment for DR at first stages of disease. Hemorrhages are the first symptoms that indicate diabetic retinopathy. Therefore, their detection is very important. In this survey paper image contrast is enhanced by preprocessing and then blood vessels are detected as the boundaries of hemorrhages are not preserved when they are in contact with blood vessels.then classification done on the basis texture feature such as area and size and standard deviation etc. finally classify the images into normal, moderate and Severe DR.
\end{abstract}

Keywords: Diabetic retinopathy, haemorrhages, Blood vessel, Diabetes.

\section{INTRODUCTION}

Diabetes is the major reason for visual loss. diabetic retinopathy occur on the retinal part of the eye. In earlier days, human experts manually identify the symptoms of diabetic retinopathy in the digital color fundus images of retina taken with the help of ophthalmoscope or fundus photography. It requires highly trained and skilled experts to perform diagnosis. But due to the increasing number of people with diabetes, detection of DR symptoms is found to be a heavy and inaccurate task while screening a large number of images. Diabetes is nothing but a disorder of metabolism. Digested food enters the body stream with the aid of a hormone called "insulin" which is produced by the pancreas. During eating, the pancreas automatically produces the correct amount of insulin. It allows glucose absorption from the blood into the cells. In individuals with diabetes, pancreas either produces too little or no insulin or the cells do not react properly to the insulin that is produced. The buildup of glucose in the blood, overflows into the urine and then passes out of the body. Therefore, the body loses its main source of fuel even though the blood contains large amounts of glucose.

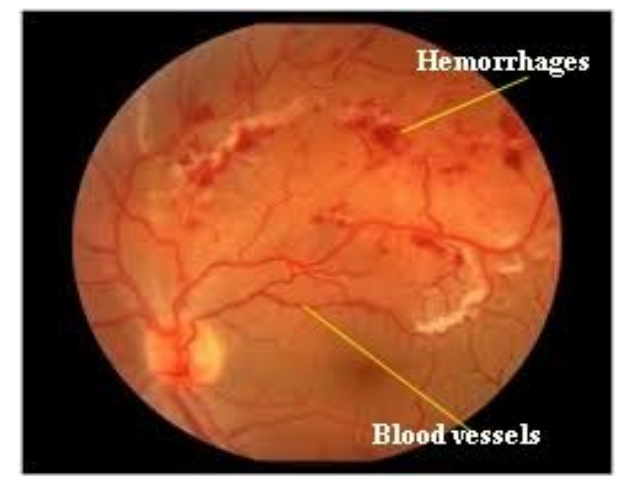

Figure 1: Diabetic retinopathy affected fundus image

Two kinds of DR are shown in Fig .The two stages of Retinopathy are Non-Proliferative and Proliferative.
Earlier stage is Non- Proliferative Diabetic Retinopathy (NPDR) in which symptoms will be mild or non-existent. Some of the Non-Proliferative Diabetic Retinopathy lesions include Micro aneurysms, Exudates and Hemorrhages. Retinal hemorrhage is useful to find NPDR. So, earlier detection of NPDR is helpful to improve automated screening system. NPDR, structural damage may occur at the back of the eye causing the blood vessels to rupture. Second, advanced or severe stage is Proliferative Diabetic Retinopathy (PDR) occurs due to new blood vessel starting to grow in the eye that are fragile and can bleed which causes Blindness. At first, the people suffering with DR may notice no changes in their vision. It could get worse over the years and threaten their good vision. Treatment for diabetic retinopathy depends on the stage of the disease and is directed.
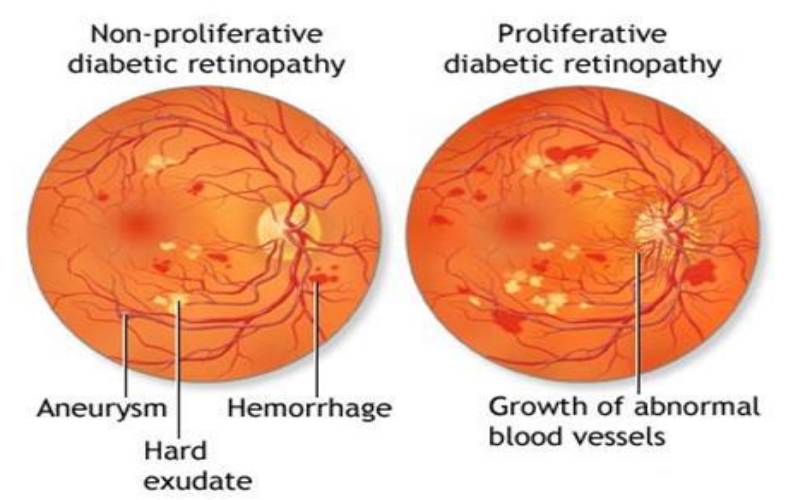

Figure 2: kinds of Diabetic Retinopathy (a) Proliferative DR.(b) Non-proliferative D

\section{LITERATURE REVIEW}

L.R.Sudha, S.Thirupurasundari, Department Of Computer Science, Annamalai University, Chidambaram published paper on "Analysis and Detection of Hemorrhages and 
exudates in retinal images" in International Journal of Scientific and Research Publications. they have proposed an automated system to detect diabetic retinopathy from retinal images. In this approach after pre-processing, texture features are extracted from retinal images to detect abnormal images. Then the abnormal images are processed to localize and identify the problem of exudates and hemorrhages.

Athira R V, PG student,Loyola institute of technology and science Kanyakumari, and Ferlin Deva Shahila D, Faculty, Loyola institute of technology and science Kanyakumari, india write paper on " Detection of Retinal Hemorrhage Using Splat Feature Classification Technique" in Int. Journal of Engineering Research and Applications. In this approach, a splat feature classification technique can be used to detect large irregular retinal hemorrhages with high accuracy. In this technique, the retinal images are partitioned into non overlapping segments called splat. Splat contains pixels with same color and spatial location. From each splat wide range of features are extracted based on interaction of each splat with its neighbor. The mean filter approach can be used to select desired splat feature. Performance of the hemorrhage detector can be evaluated from the receiver operating characteristic curve

Sumathi.P, Assistant Professor(Sr.Gr) Department of IT, SNS College of Engineering, Coimbatore, India and Sundar.R Assistant Professor Department of EEE Karpagam College of Engineering Coimbatore, India published paper on "Study of Detection of Retinal Hemorrhage in Fundus Images by Splat Feature Classification Method" in International Journal of Innovative Ideas in Research (IJIIR). Diabetic retinopathy is a disease in which the blood vessels in the retina changes or sometimes these vessels swell and leak fluid. The severity of the disease is identified using number and shape of hemorrhages. Under the supervised approach, retinal color images are partitioned into nonoverlapping segments covering the entire image. Each segment, i.e., splat, contains pixels with similar color and spatial location. A set of features is extracted from each splat. Based on the features extracted, the splats with hemorrhages are located and segmented.

Inbarathi.R, Karthikeyan.R, Dept of Computer Science \& Engineering, PSNA College of Engineering \& Technology, Dindigul, Anna University, Chennai, India. Published paper on "Detection of Retinal Hemorrhage in Fundus Images by Classifying the Splat Features Using SVM" in International Journal of Innovative Research in Science, Engineering and Technology and 2014 International Conference on Innovations in Engineering and Technology (ICIET'14) Organized by K.L.N. College of Engineering, Madurai, Tamil Nadu, India, The objective of their proposed work is to detect retinal hemorrhage for automatic screening of DR using Support Vector Machine (SVM) classifier. To detect retinal hemorrhage, retinal fundus images are taken from Messidor dataset. After pre-processing, retinal image pixel of same color and intensity, the image is partitioned into non-overlapping area that covers the entire image. Splat and GLCM feature are extracted to improve the classification accuracy. In order to classify the given input images, different classes must be represented using relevant and significant features with the help of selection method that is processed by filter and wrapper approaches. Then hemorrhage affected retina is detected by classifier. Finally classification accuracy is compared with classifier. Kanika Verma, Prakash Deep and A. G. Ramakrishnan, Senior Member, IEEE Medical Intelligence and Language Engineering Lab Department of Electrical Engineering, Indian Institute of Science, Bangalore India. published paper on topic," Detection and Classification of Diabetic Retinopathy using Retinal Images". retinal images acquired through fundal camera aid in analyzing the consequences, nature, and status of the effect of diabetes on the eye. The objectives of this study are to (i) detect blood vessel, (ii) identify haemorrhages and (iii) classify different stages of diabetic retinopathy into normal, moderate and nonproliferative diabetic retinopathy (NPDR). The basis of the classification of different stages of diabetic retinopathy is the detection and quantification of blood vessels and haemorrhages present in the retinal image. Retinal vascular is segmented utilising the contrast between the blood vessels and surrounding background. Haemorrhage candidates were detected using density analysis and bounding box techniques. Finally, classification of the different stages of eye disease was done using Random Forests technique based on the area and perimeter of the blood vessels and haemorrhages. Accuracy assessment of the classified output revealed that normal cases were classified with $90 \%$ accuracy while moderate and severe NPDR cases were $87.5 \%$ accurate.

\section{PROPOSED PLAN OF WORK}

The main goal of the proposed system is to automatically classify hemorrhages from other symptoms of DR. The input retinal images are taken from Internet which is given as input to the pre-processing.

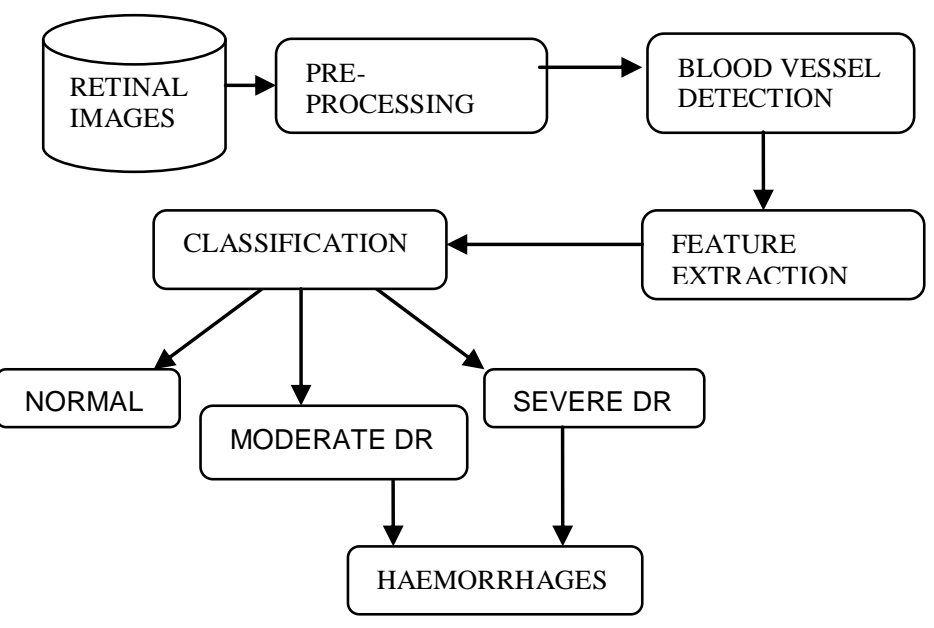

Figure 3: Block Diagram of the Proposed System 
After pre-processing, the blood vessels are detected. After detection of blood vessels extract the features from the abnormal images and then classify these retinal images does contain hemorrhages problem on the basis of feature extracted. Block diagram of the proposed system is shown in Figure 3.

Proposed system consists of four modules which are A) Pre-processing the retinal image, B) Blood Vessel Detection C) Feature Extraction and D) Classify the output

\section{A. Pre-processing}

The input of the automated system is color fundus retinal image which is taken from internet. This stage corrects the problem of illumination variation of the picture taken. The pre-processing steps consist of:

1) Resizing the retinal gray images

The input retinal images are resized into small images. It is mainly to avoid overloading and time consumption.

2) Color to green channel extraction

To convert RGB color fundus images into green channel conversion.

\section{3) Adaptive histogram technique}

Adaptive histogram is used to enhance the "contrast" and to improve the quality of retinal image. Adaptive Histogram Equalization Method (AHEM) gives better performance, higher processing speed and work well for all images are of different sizes, hence the reason for it being used as method of correcting un-even illumination. A variant of adaptive histogram equalization called Contrast Limited Adaptive Histogram Equalization (CLAHE). Images processed with CLAHE are of more natural appearance and facilitate the comparison of different areas of an image. The main objective of this method is to define a point transformation within a local fairly large window. The local window is assumed to be unaffected by the gradual variation of intensity between the image centers and edges. The point transformation distribution is localized around the mean intensity of the window and it covers the entire intensity range of the image.

\section{4) Morphological Operation}

Morphological processing is constructed with operations on sets of pixels. Binary morphology uses only set membership and is indifferent to the value, such as gray level or color, of a pixel.

The basic morphological operators are erosion, dilation, opening and closing.

Binary dilation and erosion: The set of black and white pixels constitute a description of a binary image. Assume that only black pixels are considered, and the others are treated as a background. The primary morphological operations are dilation and erosion, and from these two, more complex morphological operations such as opening, closing, and shape decomposition can be constituted.

Dilation: The dilation operation thickens the image. The extent of how much it should be thicken is based on the structuring element. The structuring element is a part of the image.

Erosion: The erosion operation performs either shrinking or thinning of the object. The extent of this operation is decided by the structuring element.

\section{B. Blood Vessel Detection}

After enhancing the contrast of the image, The designed matched filter is applied on the image to detect the blood vessels. A binarised image is obtained by thresholding. A matrix was generated to store the number of matched filter which was responsible for detecting that particular pixel of the blood vessel. The gray level value of the pixels in a particular direction of detection was multiplied by a factor. The value was then checked to be above threshold level. For $0^{\circ}, 15^{\circ}$ and $180^{\circ}$, pixels in the horizontal direction were checked; for $30^{\circ}, 45^{\circ}$ and $60^{\circ}$, pixels in the 45 degree and 225 degree directions were checked; for $75^{\circ}, 90^{\circ}$ and $105^{\circ}$, pixels in vertical direction and for $120^{\circ}, 135^{\circ}$ and $150^{\circ}$, pixels in 135 degree directions were checked. If gray value multiplied by a factor was greater than the threshold, then that pixel was counted as blood vessel. Finally, the blood vessels are extracted pixel by pixel.

\section{Feature Extraction}

In feature extraction, Texture analysis used to extract feature values from the input images. These features are used to attempts quantify qualities that are described in terms of rough, smooth and silky as a function of spatial variation are shown in pixel intensities. Texture analysis can be helpful when objects in an image are more characterized by texture than by intensity.

1) Range Filter

Range Filter is found out the local range of the gray scale images. Matlab function of range filter is used to generate ranges for the input images. It returns each output pixel that contains the range value which is greater value smaller value find for every 3-by-3 neighborhood around the corresponding pixel in the input image.

\section{2) Standard Deviation Filter}

Standard Deviation Filter calculates the local standard deviation for the input images. Standard Deviation Filter function is used from matlab which returns each output pixel contains the standard deviation of the 3-by-3 neighborhood around the corresponding pixel in the input image. The preprocessed image after the removal of optic disk and blood vessels contains only hemorrhages. This image is used for feature extraction. The statistical features extracted are hemorrhages, area, entropy, correlation, energy, contrast, homogeneity, standard deviation, mean. The extracted feature values will have different ranges of values. Thus it is necessary to normalize the values to an acceptable range. From these extracted features, effective features are selected for the classification.

\section{Classification}

We will prefer classification of hemorrhages on the basis of area and size of the pixels of image which is extracted 
during feature extraction of an image, since it provides more accuracy on a larger dataset.

\section{CONCLUSION}

The proposed automated system used to identify patients having diabetic retinopathy using fundus images. preprocessing needed for color fundus image taken from internet as it contains noise. then we will detect blood vessels as the boundaries of hemorrhage does not preserved when they are in contact with blood vessels. then we will extract texture features like standard deviation, area and size of pixels of image and apply classification on the basis of area and size of pixels and detect diabetic retinopathy diseases such as hemorrhages (red patches) which falls between normal, moderate and severe DR.

Hence, this paper presents survey and analysis of hemorrhages detection which is a primary symptoms of DR which cause visual loss.

\section{REFERENCES}

[1] Kanika Verma, Prakash Deep and A. G. Ramakrishnan, Senior Member, IEEE Medical Intelligence and Language Engineering Lab Department of Electrical Engineering, Indian Institute of Science, Bangalore India.," Detection and Classification of Diabetic Retinopathy using Retinal Images".

[2] L.R.Sudha,S.Thirupurasundari,"Analysis And Detection Of Hemorrhage And Hemorrhages In Retinal Images", International Journal of Scientific and Research Publications, Volume 4, Issue 3, March 20141 ISSN 2250-3153

[3] Inbarathi.R, Karthikeyan.R, Dept of Computer Science \& Engineering, PSNA College of Engineering \& Technology, Dindigul, Anna University, Chennai, India," Detection of Retinal Hemorrhage in Fundus Images by Classifying the Splat Features Using SVM", International Journal of Innovative Research in Science, Engineering and Technology Volume 3, Special Issue 3, March 2014

[4] Sumathi.P, Sundar.R," Study of Detection of Retinal Hemorrhage in Fundus Images by Splat Feature Classification Method", International Journal of Innovative Ideas in Research (IJIIR), Vol.1, no.1, September 2015.

[5] Athira R V, Ferlin Deva Shahila D," Detection of Retinal Hemorrhage Using Splat Feature Classification Technique”, Int. Journal of Engineering Research and Applications ISSN : 22489622, Vol. 4, Issue 1( Version 3), January 2014, pp.327-330 